\title{
Decision Wave Equation of Piezoactuator for Nanotechnology and Adaptive Optics
}

\author{
S.M. Afonin \\ Department of Intellectual Technical Systems, National Research University of Electronic Technology MIET, \\ Moscow, Russia \\ eduems@mail.ru
}

Abstract: Decision of wave equation, structural-parametric model, parametric structural schematic diagram, transfer functions of the piezoactuator are obtained using the Laplace transform. Effects of geometric and physical parameters of the piezoactuator and the external load on its dynamic characteristics are determined. The transfer functions and the parametric structural schematic diagram of the piezoactuator for the transverse, longitudinal, shift piezoelectric effects are obtained from the structural-parametric model of the piezoactuator. For calculation of the control systems for nanotechnology with the piezoactuator its the parametric structural schematic diagram and the transfer functions are determined. The generalized structural-parametric model and the generalized parametric structural schematic diagram of the piezoactuator for nanotechnology and adaptive optics are constructed.

Keywords: decision wave equations, piezoactuator, nanotechnology, deformation, nano- and microdisplacement, structural-parametric model, parametric structural schematic diagram, transfer functions.

\section{INTRODUCTION}

In nanotechnology and adaptive optics the piezoelectric actuator (piezoactuator) solves problem of precise alignment, compensation of temperature and gravitational deformations. Piezoactuator for mechatronic systems from piezoceramics PZT is promising in nanotechnology, nanotechnology, nanobiotechnology, microelectronics, power engineering, astronomy for large compound telescopes, antennas satellite telescopes and adaptive optics equipment for compensation of atmospheric turbulence by wave front correction. Piezoactuator - piezomechanical device intended for actuation of mechanisms, systems or management based on the piezoelectric effect, converts electrical signals into mechanical movement and force [1 - 9].

In the present article is solving the problem of building the structural parametric model of the piezoactuator for nanotechnology and adaptive optics in contrast electrical equivalent circuits Cady-Mason for calculation of piezoelectric transmitter and receiver [10 - 15]. Consider building the structural-parametric model of the piezoactuator, representing the system of equations, which, given the electromechanical parameters of the piezoactuator describes the structure and conversion the energy electric field into mechanical energy and the corresponding displacements and forces at its the ends. By solving the wave equation with allowance methods of mathematical physics using the Laplace transform for the corresponding equation of piezoelectric effect, the boundary conditions on loaded working surfaces of the piezoactuator, the strains along the coordinate axes, it is possible to construct a structural-parametric model of the piezoactuator. The transfer functions and the parametric structural schematic diagrams of the piezoactuator are obtained from its structural-parametric model [16 - 34].

The piezoactuator of nano- and micrometric movements operates based on the inverse piezoeffect, in which the motion is achieved due to deformation of the piezoelement when an external electric voltage is applied to it. Piezoactuator for drives of nano- and micrometric movements provide a movement range from several 
nanometers to tens of micrometers, a sensitivity of up to $10 \mathrm{~nm} / \mathrm{V}$, a loading capacity of up to $1000 \mathrm{~N}$, a ransmission band of up to $1000 \mathrm{~Hz}$. The investigation of static and dynamic characteristics of a piezoactuator is necessary for calculation mechatronic systems of nanotechnology and adaptive optics. Piezoactuator provide high stress and speed of operation and return to the initial state when switched off. Piezoactuator for precise alignment is used in the majority mechatronic systems of scanning tunneling microscopes (STMs), scanning force microscopes (SFMs), atomic force microscopes (AFMs) [16 - 36].

\section{Decision Wave Equation and Structural-Parametric Model of Piezoactuator}

Deformation of the piezoactuator corresponds to its stressed state, therefore in the piezoactuator there are six stress components $T_{1}, T_{2}, T_{3}, T_{4}, T_{5}, T_{6}$, the components $T_{1}-T_{3}$ are related to extension-compression stresses, $T_{4}-T_{6}$ to shear stresses.

The matrix state equations [12] connecting the electric and elastic variables for polarized piezoceramics have the following form:

$$
\begin{gathered}
\mathbf{D}=\mathbf{d} \mathbf{T}+\boldsymbol{\varepsilon}^{T} \mathbf{E}, \\
\mathbf{S}=\mathbf{s}^{E} \mathbf{T}+\mathbf{d}^{t} \mathbf{E},
\end{gathered}
$$

where the first equation describes the direct piezoelectric effect, and the second - the inverse piezoelectric effect; $\mathbf{D}$ is the column matrix of electric induction along the coordinate axes; $\mathbf{S}$ is the column matrix of relative deformations; $\mathbf{T}$ is the column matrix of mechanical stresses; $\mathbf{E}$ is the column matrix of electric field strength along the coordinate axes; $\mathbf{s}^{E}$ is the elastic compliance matrix for $E=$ const; $\boldsymbol{\varepsilon}^{T}$ is the matrix of dielectric constants for $T=$ const ; $\mathbf{d}^{t}$ is the the transposed matrix of the piezoelectric modules.

In polarized piezoceramics from lead zirconate titanate PZT for the piezoactuator for nanotechnology and adaptive optics there are five independent components $s_{11}^{E}, s_{12}^{E}, s_{13}^{E}, s_{33}^{E}, s_{55}^{E}$ in the elastic compliance matrix, three independent components $d_{33}, d_{31}, d_{15}$ in the transposed matrix of the piezoelectric modules and three independent components $\varepsilon_{11}^{T}, \varepsilon_{22}^{T}, \varepsilon_{33}^{T}$ in the matrix of dielectric constants. Let us consider the simplest piezoactuator in Figure 1 for longitudinal, transverse and shift deformations.

Let us consider the piezoactuator for the longitudinal piezoelectric effect, where $\delta$ is thickness and the electrodes deposited on its faces perpendicular to axis 3. the area of which is equal to $S_{0}$. The direction of the polarization axis $P$, i.e., the direction along which polarization was performed, is usually taken as the direction of axis 3 . The equation of the inverse longitudinal piezoelectric effect $[12,15,16]$ has the form:

$$
S_{3}=d_{33} E_{3}(t)+s_{33}^{E} T_{3}(x, t),
$$

where $S_{3}=\partial \xi(x, t) / \partial x$ is the relative displacement of the cross section of the piezoactuator, $d_{33}$ is the piezomodule for the longitudinal piezoeffect, $E_{3}(t)=U(t) / \delta$ is the electric field strength, $U(t)$ is the voltage between the electrodes of actuator, $\delta$ is the thickness, $s_{3}^{E}$ is the elastic compliance along axis 3 , and $T_{3}$ is the mechanical stress along axis 3.

The equation of equilibrium for the forces acting on the piezoactuator (piezoelectric plate) on Figure 1 can be written as

$$
T_{3} S_{0}=F+M \frac{\partial^{2} \xi(x, t)}{\partial t^{2}}
$$

where $F$ is the external force applied to the piezoactuator, $S_{0}$ is the cross section area and $M$ is the displaced mass. 


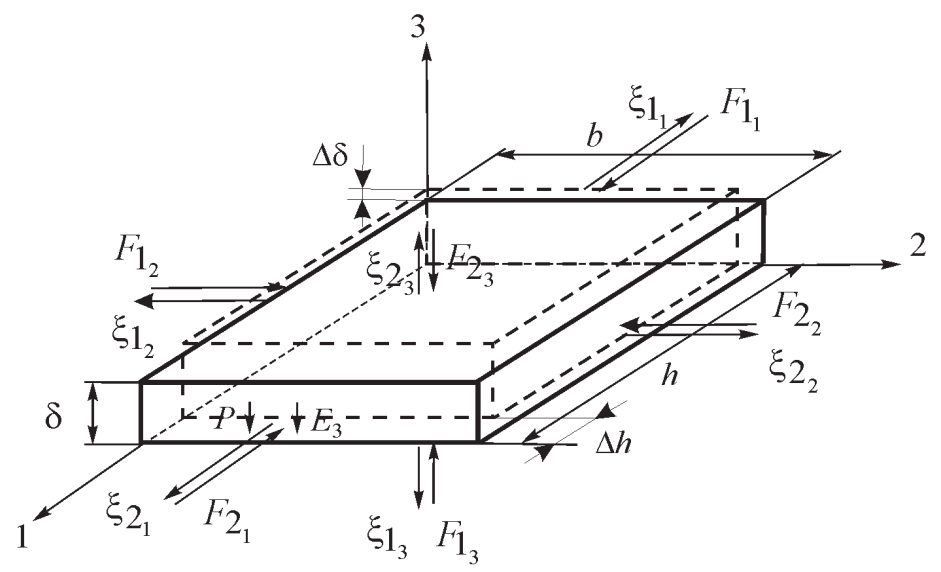

Figure1. Piezoactuator.

For constructing a structural parametric model of the voltage-controlled piezoactuator, let us solve simultaneously the wave equation, the equation of the inverse longitudinal piezoeffect, the equation of forces acting on the faces of the piezoactuator.

Calculations of the piezoactuators are performed using a wave equation $[6,12,15,16]$ describing the wave propagation in a long line with damping but without distortions, which can be written in the form:

$$
\frac{1}{\left(c^{E}\right)^{2}} \frac{\partial^{2} \xi(x, t)}{\partial t^{2}}+\frac{2 \alpha}{c^{E}} \frac{\partial \xi(x, t)}{\partial t}+\alpha^{2} \xi(x, t)=\frac{\partial^{2} \xi(x, t)}{\partial x^{2}},
$$

where $\xi(x, t)$ is the displacement of the section, $x$ is the coordinate, $t$ is time, $c^{E}$ is the sound speed for $E=$ const $\alpha$ is the damping coefficient.

Using the Laplace transform, we can reduce the original problem for the partial differential hyperbolic equation of type (5) to a simpler problem for the linear ordinary differential equation [14, 15, 16].

Applying the Laplace transform to the wave equation (5)

$$
\Xi(x, p)=L\left\{\xi(x, t\}=\int_{0}^{\infty} \xi(x, t) e^{-p t} d t\right.
$$

and setting the zero initial conditions, we obtain the linear ordinary second-order differential equation with the parameter $\mathrm{p}$

$$
\frac{d^{2} \Xi(x, p)}{d x^{2}}-\left[\frac{1}{\left(c^{E}\right)^{2}} p^{2}+\frac{2 \alpha}{c^{E}} p+\alpha^{2}\right] \Xi(x, p)=0,
$$

with its solution being the function

$$
\Xi(x, p)=C e^{-x_{\gamma}}+B e^{x_{Y}},
$$

where $\Xi(x, p)$ is the Laplace transform of the displacement of the section of the piezoelectric actuator, $\gamma=p / c^{E}+\alpha$ is the propagation coefficient. Determining coefficients $C$ and $B$ as

$$
\begin{array}{ll}
\Xi(0, p)=\Xi_{1}(p) & \text { for } x=0, \\
\Xi(\delta, p)=\Xi_{2}(p) & \text { for } x=\delta .
\end{array}
$$

Then, the coefficients $C$ and $B$ in the form 


$$
C=\left(\Xi_{1} e^{\delta \gamma}-\Xi_{2}\right) /[2 \operatorname{sh}(\delta \gamma)], B=\left(\Xi_{1} e^{-\delta \gamma}-\Xi_{2}\right) /[2 \operatorname{sh}(\delta \gamma)] .
$$

The solution (7) can be written as

$$
\Xi(x, p)=\left\{\Xi_{1}(p) \operatorname{sh}[(\delta-x) \gamma]+\Xi_{2}(p) \operatorname{sh}(x \gamma)\right\} / \operatorname{sh}(\delta \gamma) .
$$

The equations for the forces on the faces of the piezoactuator

$$
\begin{gathered}
T_{3}(0, p) S_{0}=F_{1}(p)+M_{1} p^{2} \Xi_{1}(p) \text { for } x=0, \\
T_{3}(\delta, p) S_{0}=-F_{2}(p)+M_{2} p^{2} \Xi_{1}(p) \text { for } x=\delta
\end{gathered}
$$

where $T_{3}(0, p)$ and $T_{3}(\delta, p)$ are determined from the equation of the inverse piezoelectric effect.

For $x=0$ and $x=\delta$, we obtain the set of equations for determining stresses in the piezoactuator $[15,16]$ :

$$
\begin{aligned}
& T_{3}(0, p)=\left.\frac{1}{s_{33}^{E}} \frac{d \Xi(x, p)}{d x}\right|_{x=0}-\frac{d_{33}}{s_{33}^{E}} E_{3}(p), \\
& T_{3}(\delta, p)=\left.\frac{1}{s_{33}^{E}} \frac{d \Xi(x, p)}{d x}\right|_{x=5}-\frac{d_{33}}{s_{33}^{E}} E_{3}(p) .
\end{aligned}
$$

The set of equations (13) yield the set of equations for the structural-parametric model of the piezoactuator and parametric structural schematic diagram of a voltage-controlled piezoactuator for longitudinal piezoelectric effect on Figure 2:

$$
\begin{aligned}
& \Xi_{1}(p)=\left[1 /\left(M_{1} p^{2}\right)\right]\left\{-F_{1}(p)+\left(1 / \chi_{33}^{E}\right)\left[d_{33} E_{3}(p)-[\gamma / \operatorname{sh}(\delta \gamma)]\left[\operatorname{ch}(\delta \gamma) \Xi_{1}(p)-\Xi_{2}(p)\right]\right]\right\}, \\
& \left.\left.\Xi_{2}(p)=\left[1 /\left(M_{2} p^{2}\right)\right]\right\}-F_{2}(p)+\left(1 / \chi_{33}^{E}\right)\left[d_{33} E_{3}(p)-[\gamma / \operatorname{sh}(\delta \gamma)]\left[\operatorname{ch}(\delta \gamma) \Xi_{2}(p)-\Xi_{1}(p)\right]\right]\right\},
\end{aligned}
$$

where $\cdot \chi_{33}^{E}=s_{33}^{E} / s_{0}$.

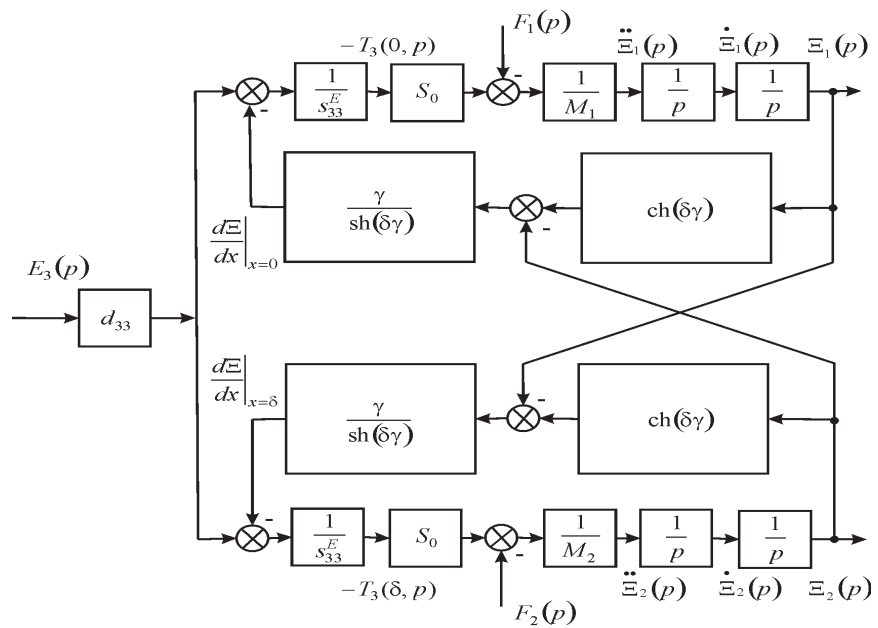

Figure2. Parametric structural schematic diagram of a voltage-controlled piezoactuator for longitudinal piezoelectric effect. 
Decision Wave Equation of Piezoactuator for Nanotechnology and Adaptive Optics

The equation of the inverse transverse piezoeffect $[7,12,16]$

$$
S_{1}=d_{31} E_{3}(t)+s_{11}^{E} T_{1}(x, t)
$$

where $S_{1}=\partial \xi(x, t) / \partial x$ is the relative displacement of the cross section along axis $1, d_{31}$ is the piezoelectric module for the transverse piezoeffect, $s_{11}^{E}$ is the elastic compliance along axis $1, T_{1}$ is the stress along axis 1.

The solution of the linear ordinary differential equation (7) can be written as (8), where the constants C and B

$$
\begin{aligned}
& \Xi(0, p)=\Xi_{1}(p) \text { for } x=0, \\
& \Xi(h, p)=\Xi_{2}(p) \text { for } x=h, \\
& \quad C=\left(\Xi_{1} e^{h \gamma}-\Xi_{2}\right) /[2 \operatorname{sh}(h \gamma)], B=\left(\Xi_{1} e^{-h \gamma}-\Xi_{2}\right) /[2 \operatorname{sh}(h \gamma)]
\end{aligned}
$$

Then, the solution (7) can be written as

$$
\Xi(x, p)=\left\{\Xi_{1}(p) \operatorname{sh}[(h-x) \gamma]+\Xi_{2}(p) \operatorname{sh}(x \gamma)\right\} / \operatorname{sh}(h \gamma)
$$

The equations of forces acting on the faces of the piezoactuator

$$
\begin{gathered}
T_{1}(0, p) S_{0}=F_{1}(p)+M_{1} p^{2} \Xi_{1}(p) \quad \text { for } x=0, \\
T_{1}(h, p) S_{0}=-F_{2}(p)+M_{2} p^{2} \Xi_{2}(p) \text { for } x=h,
\end{gathered}
$$

where $T_{1}(0, p), T_{1}(h, p)$ are determined from the equation of the inverse piezoeffect $[15,16]$. Thus, we obtain

$$
\begin{aligned}
& T_{1}(0, p)=\left.\frac{1}{s_{11}^{E}} \frac{d \Xi(x, p)}{d x}\right|_{x=0}-\frac{d_{31}}{s_{11}^{E}} E_{3}(p)^{\prime} \\
& T_{1}(h, p)=\left.\frac{1}{s_{11}^{E}} \frac{d \Xi(x, p)}{d x}\right|_{x=h}-\frac{d_{31}}{s_{11}^{E}} E_{3}(p),
\end{aligned}
$$

The set of equations (20) for stresses in piezoactuator yields the following set of equations describing the structural-parametric model and parametric structural schematic diagram of piezoactuator Figure 3

$$
\begin{aligned}
& \Xi_{1}(p)=\left[1 /\left(M_{1} p^{2}\right)\right]\left\{-F_{1}(p)+\left(1 / \chi_{11}^{E}\right)\left[d_{31} E_{3}(p)-[\gamma / \operatorname{sh}(h \gamma)]\left[\operatorname{ch}(h \gamma) \Xi_{1}(p)-\Xi_{1}(p)\right]\right]\right\}, \\
& \Xi_{2}(p)=\left[1 /\left(M_{2} p^{2}\right)\right]\left\{-F_{2}(p)+\left(1 / \chi_{11}^{E}\right)\left[d_{31} E_{3}(p)-[\gamma / \operatorname{sh}(h \gamma)]\left[\operatorname{ch}(h \gamma) \Xi_{2}(p)-\Xi_{1}(p)\right]\right]\right\},
\end{aligned}
$$

where. $\chi_{11}^{E}=s_{11}^{E} / s_{0}$. 


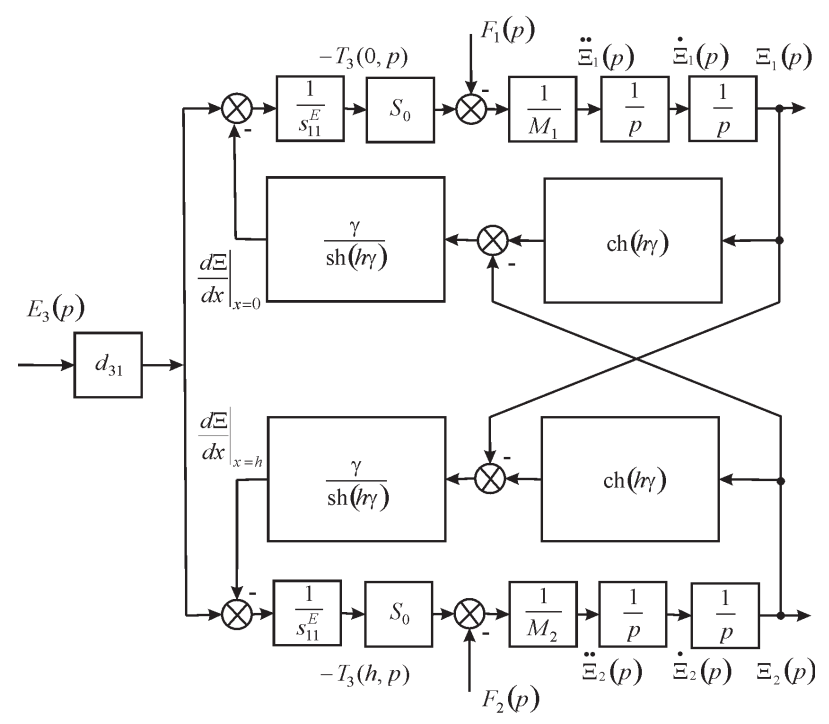

Figure3. Parametric structural schematic diagram of a voltage-controlled piezoactuator for transverse piezoelectric effect.

Let us consider the piezoactuator for the shift piezoelectric effect.

The equation of the inverse shift piezoeffect $[7,12,16]$

$$
S_{5}=d_{15} E_{1}(t)+s_{55}^{E} T_{5}(x, t)
$$

where $S_{5}=\partial \xi(x, t) / \partial x$ is the relative shift displacement, $d_{15}$ is the piezoelectric module for the shift piezoeffect, $s_{55}^{E}$ is the shift elastic compliance, $T_{5}$ is the shitt stress.

We obtain the following set of equations describing the structural-parametric model and parametric structural schematic diagram of piezoactuator Figure 4

$$
\begin{aligned}
& \Xi_{1}(p)=\left[1 /\left(M_{1} p^{2}\right)\right]\left\{-F_{1}(p)+\left(1 / \chi_{55}^{E}\right)\left[d_{15} E_{1}(p)-[\gamma / \operatorname{sh}(b \gamma)]\left[\operatorname{ch}(b \gamma) \Xi_{1}(p)-\Xi_{1}(p)\right]\right],\right. \\
& \Xi_{2}(p)=\left[1 /\left(M_{2} p^{2}\right)\right]\left\{-F_{2}(p)+\left(1 / \chi_{55}^{E}\right)\left[d_{15} E_{1}(p)-[\gamma / \operatorname{sh}(b \gamma)]\left[\operatorname{ch}(b \gamma) \Xi_{2}(p)-\Xi_{1}(p)\right]\right]\right\},
\end{aligned}
$$

where $\cdot \chi_{55}^{E}=s_{55}^{E} / s_{0}$. 


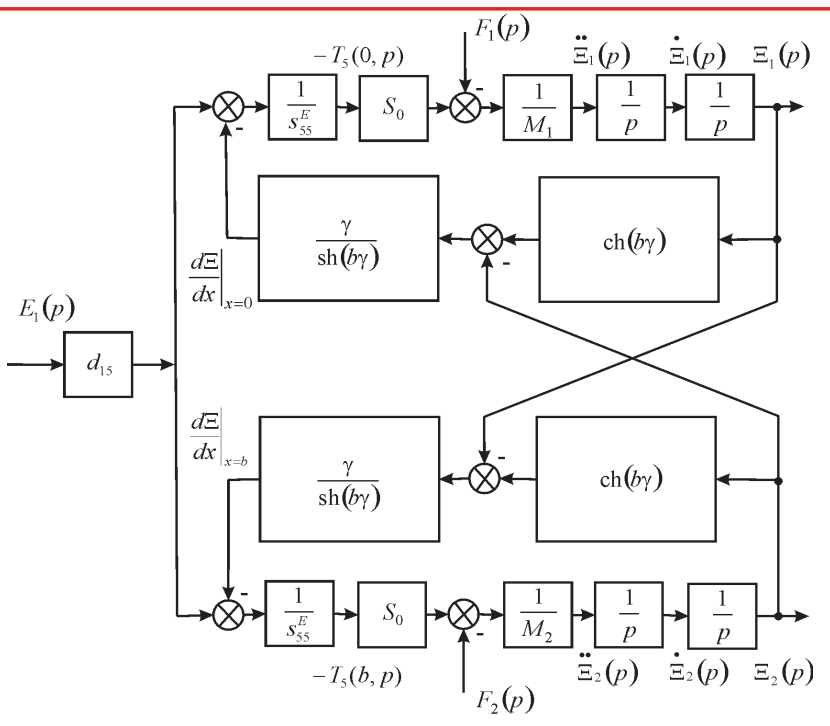

Figure4. Parametric structural schematic diagram of a voltage-controlled piezoactuator for shift piezoelectric effect.

From (14), (21), (23) we obtain the system of equations describing the generalized structural-parametric model of the piezoactuator for nanotechnology and adaptive optics in the form

$$
\begin{aligned}
& \Xi_{1}(p)=\left[1 /\left(M_{1} p^{2}\right)\right]\left\{-F_{1}(p)+\left(1 / \chi_{i j}^{\Psi}\right)\left[d_{m i} \Psi_{m}(p)-[\gamma / \operatorname{sh}(l \gamma)]\left[\operatorname{ch}(l \gamma) \Xi_{1}(p)-\Xi_{2}(p)\right]\right\},\right. \\
& \Xi_{2}(p)=\left[1 /\left(M_{2} p^{2}\right)\right]\left\{-F_{2}(p)+\left(1 / \chi_{i j}^{\Psi}\right)\left[d_{m i} \Psi_{m}(p)-[\gamma / \operatorname{sh}(l \gamma)]\left[\operatorname{ch}(l \gamma) \Xi_{2}(p)-\Xi_{1}(p)\right]\right]\right\}, \\
& \qquad d_{m i}=\left\{\begin{array}{l}
d_{33}, d_{31}, d_{15} \\
g_{33}, g_{31}, g_{15}
\end{array}, \Psi_{m}=\left\{\begin{array}{l}
E_{3}, E_{3}, E_{1} \\
D_{3}, D_{3}, D_{1}
\end{array}, s_{i j}^{\Psi}=\left\{\begin{array}{c}
s_{33}^{E}, s_{11}^{E}, s_{55}^{E} \\
s_{33}^{D}, s_{11}^{D}, s_{55}^{D}
\end{array}, l=\left\{\delta, h, b, c^{\Psi}=\left\{c^{E}, c^{D}, \gamma^{\Psi}=\left\{\gamma^{E}, \gamma^{D},\right.\right.\right.\right.\right.\right.
\end{aligned}
$$

where

$$
\chi_{i j}^{\Psi}=s_{i j}^{\Psi} / s_{0},
$$

then parameter $\Psi$ of the control parameter for the piezoactuator: $E$ for voltage control, $D$ for current control. On Figure 5 is shown the generalized parametric structural schematic diagram of piezoactuator corresponding to the set of equations (24). 


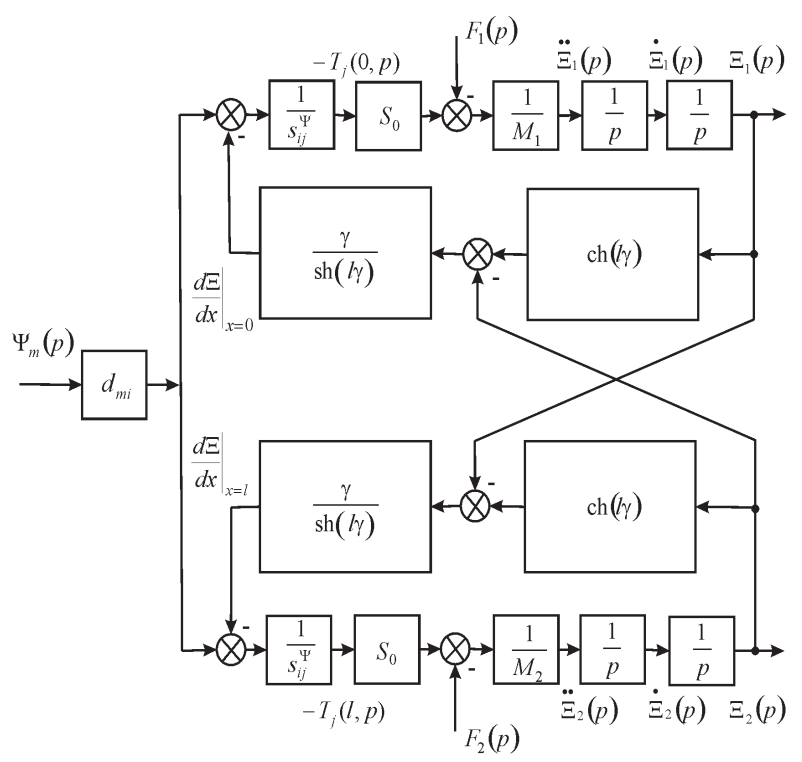

Figure5. Generalized parametric structural schematic diagram of the piezoactuator.

\section{Transfer Functions of Piezoactuator}

From generalized structural-parametric model (24) of the piezoactuator after algebraic transformations we obtain the transfer functions in matrix form [15 - 34], where the transfer functions are the ratio of the Laplace transform of the displacement of the face piezoactuator and the Laplace transform of the corresponding control parameter or force at zero initial conditions.

$$
\begin{aligned}
& \Xi_{1}(p)=W_{11}(p) \Psi_{m}(p)+W_{12}(p) F_{1}(p)+W_{13}(p) F_{2}(p), \\
& \Xi_{2}(p)=W_{21}(p) \Psi_{m}(p)+W_{22}(p) F_{1}(p)+W_{23}(p) F_{2}(p),
\end{aligned}
$$

where the generalized transfer functions of the piezoactuator are

$$
\begin{gathered}
W_{11}(p)=\Xi_{1}(p) / \Psi_{m}(p)=d_{m i}\left[M_{2} \chi_{i j}^{\Psi} p^{2}+\gamma \operatorname{th}(l \gamma / 2)\right] / A_{i j}, \\
A_{i j}=M_{1} M_{2}\left(\chi_{i j}^{\Psi}\right)^{2} p^{4}+\left\{\left(M_{1}+M_{2}\right) \chi_{i j}^{\Psi} /\left[c^{\Psi} \operatorname{th}(l \gamma)\right]\right\} p^{3}+\left[\left(M_{1}+M_{2}\right) \chi_{i j}^{\Psi} \alpha / \operatorname{th}(l \gamma)+1 /\left(c^{\Psi}\right)^{2}\right] p^{2}+2 \alpha p / c^{\Psi}+\alpha^{2}, \\
W_{21}(p)=\Xi_{2}(p) / \Psi_{m}(p)=d_{m i}\left[M_{1} \chi_{i j}^{\Psi} p^{2}+\gamma \operatorname{th}(l \gamma / 2)\right] / A_{i j}, \\
W_{12}(p)=\Xi_{1}(p) / F_{1}(p)=-\chi_{i j}^{\Psi}\left[M_{2} \chi_{i j}^{\Psi} p^{2}+\gamma / \operatorname{th}(l \gamma)\right] / A_{i j}, \\
W_{13}(p)=\Xi_{1}(p) / F_{2}(p)=W_{22}(p)=\Xi_{2}(p) / F_{1}(p)=\left[\chi_{i j}^{\Psi} \gamma / \operatorname{sh}(l \gamma)\right] / A_{i j},
\end{gathered}
$$


Decision Wave Equation of Piezoactuator for Nanotechnology and Adaptive Optics

$$
W_{23}(p)=\Xi_{2}(p) / F_{2}(p)=-\chi_{i j}^{\Psi}\left[M_{1} \chi_{i j}^{\Psi} p^{2}+\gamma / \operatorname{th}(l \gamma)\right] / A_{i j} .
$$

Therefore, we obtain from equations (25) the generalized matrix equation for the piezoactuator

$$
\left(\begin{array}{l}
\Xi_{1}(p) \\
\Xi_{2}(p)
\end{array}\right)=\left(\begin{array}{lll}
W_{11}(p) & W_{12}(p) & W_{13}(p) \\
W_{21}(p) & W_{22}(p) & W_{23}(p)
\end{array}\right)\left(\begin{array}{c}
\Psi_{m}(p) \\
F_{1}(p) \\
F_{2}(p)
\end{array}\right)
$$

Let us find the displacement of the faces the piezoactuator in a stationary regime for $\Psi_{m}(t)=\Psi_{m 0} \cdot 1(t)$, $F_{1}(t)=F_{2}(t)=0$ and inertial load. The static displacement of the faces the piezoactuator $\xi_{1}(\infty)$ and $\xi_{2}(\infty)$ can be written in the form:

$$
\begin{gathered}
\xi_{1}(\infty)=\lim _{t \rightarrow \infty} \xi_{1}(t)=\lim _{\substack{p \rightarrow 0 \\
\alpha \rightarrow 0}} p W_{11}(p) \Psi_{m 0} / p=d_{m i} l \Psi_{m 0}\left(M_{2}+m / 2\right) /\left(M_{1}+M_{2}+m\right), \\
\xi_{2}(\infty)=\lim _{t \rightarrow \infty} \xi_{2}(t)=\lim _{\substack{p \rightarrow 0 \\
\alpha \rightarrow 0}} p W_{21}(p) \Psi_{m 0} / p=d_{m i} l \Psi_{m 0}\left(M_{1}+m / 2\right) /\left(M_{1}+M_{2}+m\right), \\
\xi_{1}(\infty)+\xi_{2}(\infty)=\lim _{t \rightarrow \infty}\left(\xi_{1}(t)+\xi_{2}(t)\right)=d_{m i} l \Psi_{m 0},
\end{gathered}
$$

where $m$ is the mass of the piezoactuator, $M_{1}, M_{2}$ are the load masses.

Let us consider a numerical example of the calculation of static characteristics of the piezoactuator from piezoceramics PZT under the longitudinal piezoelectric effect at $m \ll M_{1}$, and $m \ll M_{2}$ 'or $d_{33}=4 \cdot 10^{-10} \mathrm{~m} / \mathrm{V}$, $U=375 \mathrm{~V}, \quad M_{1}=10 \mathrm{~kg}$ and $\quad M_{2}=40 \mathrm{~kg}$ we obtain the static displacement of the faces of the piezoactuator $\xi_{1}(\infty)=120 \mathrm{~nm}, \xi_{2}(\infty)=30 \mathrm{~nm}, \xi_{1}(\infty)+\xi_{2}(\infty)=150 \mathrm{~nm}$.

The static displacement the faces of the piezoactuator for the transverse piezoelectric effect and inertial load at $U(t)=U_{0} \cdot 1(t), E_{3}(t)=E_{30} \cdot 1(t)=\left(U_{0} / \delta\right) \cdot 1(t)$ and $F_{1}(t)=F_{2}(t)=0$ can be written in the following form:

$$
\begin{aligned}
& \xi_{1}(\infty)= \lim _{t \rightarrow \infty} \xi_{1}(t)=\lim _{\substack{p \rightarrow 0 \\
\alpha \rightarrow 0}} p W_{11}(p)\left(U_{0} / \delta\right) / p=d_{31}(h / \delta) U_{0}\left(M_{2}+m / 2\right) /\left(M_{1}+M_{2}+m\right), \\
& \xi_{2}(\infty)=\lim _{t \rightarrow \infty} \xi_{2}(t)=\lim _{\substack{p \rightarrow 0 \\
\alpha \rightarrow 0}} p W_{21}(p)\left(U_{0} / \delta\right) / p=d_{31}(h / \delta) U_{0}\left(M_{1}+m / 2\right) /\left(M_{1}+M_{2}+m\right), \\
& \xi_{1}(\infty)+\xi_{2}(\infty)=\lim _{t \rightarrow \infty}\left(\xi_{1}(t)+\xi_{2}(t)\right)=d_{31}(h / \delta) U_{0} .
\end{aligned}
$$

The static displacement of the faces of the piezoactuator for the transverse piezoeffect at, $m \ll M_{1}$, $m \ll M_{2}$

$$
\xi_{1}(\infty)=\lim _{t \rightarrow \infty} \xi_{1}(t)=\lim _{\substack{p \rightarrow 0 \\ \alpha \rightarrow 0}} p W_{11}(p)\left(U_{0} / \delta\right) / p=d_{31}(h / \delta) U_{0} M_{2} /\left(M_{1}+M_{2}\right),
$$




$$
\xi_{2}(\infty)=\lim _{t \rightarrow \infty} \xi_{2}(t)=\lim _{\substack{p \rightarrow 0 \\ \alpha \rightarrow 0}} p W_{21}(p)\left(U_{0} / \delta\right) / p=d_{31}(h / \delta) U_{0} M_{1} /\left(M_{1}+M_{2}\right) .
$$

Let us consider a numerical example of the calculation of static characteristics of the piezoactuator from piezoceramics PZT under the transverse piezoelectric effect at $m \ll M_{1}$ and $m \ll M_{2}$. For $d_{31}=2.5 \cdot 10^{-10} \mathrm{~m} / \mathrm{V}$, $h=4 \cdot 10^{-2} \mathrm{~m}, \delta=2 \cdot 10^{-3} \mathrm{~m}, U=300 \mathrm{~V}, M_{1}=10 \mathrm{~kg}$ and $M_{2}=40 \mathrm{~kg}$ we obtain the static displacement of the faces of the piezoelectric actuator $\xi_{1}(\infty)=1200 \mathrm{~nm}, \xi_{2}(\infty)=300 \mathrm{~nm}, \xi_{1}(\infty)+\xi_{2}(\infty)=1500 \mathrm{~nm}$.

For the description of the piezoactuator for the longitudinal piezoelectric effect for one rigidly fixed face of the transducer at $M_{1} \rightarrow \infty, R=0$, where $R$ is the resistance of the circuit, we obtain from equation (25) the transfer functions $W_{21}(p)$ and $W_{23}(p)$ of the piezoactuator for the longitudinal piezoelectric effect in the following form:

$$
\begin{aligned}
& W_{21}(p)=\Xi_{2}(p) / E_{3}(p)=d_{33} \delta /\left[M_{2} \delta \chi_{33}^{E} p^{2}+\delta \gamma \operatorname{cth}(\delta \gamma)\right] \\
& W_{23}(p)=\Xi_{2}(p) / F_{2}(p)=-\delta \chi{ }_{33}^{E} /\left[M_{2} \delta \chi{ }_{33}^{E} p^{2}+\delta \gamma \operatorname{cth}(\delta \gamma)\right] .
\end{aligned}
$$

We obtain the transfer functions of the piezoactuator for the transverse piezoelectric effect for one rigidly fixed face of the piezoactuator at $M_{1} \rightarrow \infty$ in the form:

$$
\begin{aligned}
& W_{21}(p)=\Xi_{2}(p) / E_{3}(p)=d_{31} h /\left[M_{2} h \chi_{11}^{E} p^{2}+h \gamma \operatorname{cth}(h \gamma)\right], \\
& W_{23}(p)=\Xi_{2}(p) / F_{2}(p)=-h \chi_{11}^{E} /\left[M_{2} h \chi_{11}^{E} p^{2}+l \gamma \operatorname{cth}(l \gamma)\right] .
\end{aligned}
$$

For $M_{1} \rightarrow \infty, \alpha=0$ and $M_{2}=0$ we write the resonance condition

$$
\operatorname{ctg} k h=\mathrm{o}
$$

where $k=\omega / c^{E}$ is frequency coefficient; $\omega$ is circular frequency.

Therefore

$$
k_{i} h=\pi(2 i-1) / 2
$$

where index $i=1,2,3, \ldots$.

This means that the piezoactuator is a quarter-wave vibrator with the resonance frequency

$$
f_{1}=c^{E} /(4 h) \text {. }
$$

For the piezoactuator for the transverse piezoelectric effect at $c^{E}=3 \cdot 10^{3} \mathrm{~m} / \mathrm{s}, h=4 \cdot 10^{-2} \mathrm{~m}$ is obtained the resonance frequency $f_{1}=18,75 \mathrm{kHz}$. The experimental and calculated values for the piezoactuator are in agreement up to an accuracy of $5 \%$.

Accordingly, the static displacement $\xi_{2}(\infty)$ of the piezoactuator under the longitudinal piezoeffect in the form:

$$
\begin{gathered}
\xi_{2}(\infty)=\lim _{t \rightarrow \infty} \xi_{2}(t)=\lim _{p \rightarrow 0} p W_{2}(p) U_{0} / p=d_{33} U_{0}, \\
\xi_{2}(\infty)=\lim _{p \rightarrow 0} p W_{23}(p) F_{0} / p=-\delta s_{33}^{E} F_{0} / S_{0}
\end{gathered}
$$

Let us consider the calculation of static characteristics of the piezoactuator from piezoceramics PZT under the longitudinal piezoeffects. For $d_{33}=5 \cdot 10^{-10} \mathrm{~m} / \mathrm{V}, U=120 \mathrm{~V}$ we obtain $\xi_{2}(\infty)=60 \mathrm{~nm}$. For $\delta=6 \cdot 10^{-4} \mathrm{~m}$, 
$s_{33}^{E}=3.5 \cdot 10^{-11} \mathrm{~m}^{2} / \mathrm{N}, F_{0}=500 \mathrm{~N}, S_{0}=1.75 \cdot 10^{-4}: \mathrm{m}^{2}$ we received $\xi_{2}(\infty)=-60 \mathrm{~nm}$.

Let us consider the operation at low frequencies for the piezoactuator with one face rigidly fixed so that $M_{1} \rightarrow \infty$ and $m \ll M_{2}$. Using the approximation of the hyperbolic cotangent by two terms of the power series in transfer functions (35) and (36), at $m \ll M_{2}$ we obtain the expressions in the frequency range of $0<\omega<0,01 c^{E} / \delta$

$$
\begin{gathered}
W_{21}(p)=\Xi_{2}(p) / E_{3}(p)=d_{33} \delta /\left(T_{t}^{2} p^{2}+2 T_{t} \xi_{t} p+1\right) \\
W_{23}(p)=\Xi_{2}(p) / F_{2}(p)=-\left(s_{33}^{E} \delta / S_{0}\right) /\left(T_{t}^{2} p^{2}+2 T_{t} \xi_{t} p+1\right) \\
T_{t}=\left(\delta / c^{E}\right) \sqrt{M_{2} / m}=\sqrt{M_{2} / C_{33}^{E}}, \xi_{t}=(\alpha \delta / 3) \sqrt{m / M_{2}}, C_{33}^{E}=S_{0} /\left(s_{33}^{E} \delta\right)=1 /\left(\chi_{33}^{E} \delta\right) .
\end{gathered}
$$

where $T_{t}$ is the time constant and $\xi_{t}$ is the damping coefficient, $C_{33}^{E}$ - is the is rigidity of the piezoactuator under the longitudinal piezoeffect.

In the static mode of operation the piezoactuator for elastic load we obtain the equation the following form

$$
\frac{\xi_{2}}{\xi_{2 m}}=\frac{1}{1+C_{e} / C_{33}^{E}}
$$

where $\xi_{2}$ is the displacement of the piezoactuator in the case of the elastic load, $\xi_{2 m}=d_{33} U_{0}$ is the maximum displacement of the piezoactuator, $C_{e}$ is the load rigidity.

From (44), (46) we obtain the transfer functions of the piezoactuator with a fixed end and elastic inertial load

$$
W_{2}(p)=\frac{\Xi_{2}(p)}{U(p)}=\frac{d_{33}}{\left(1+C_{e} / C_{33}^{E}\right)\left(T_{t}^{2} p^{2}+2 T_{t} \xi_{t} p+1\right)},
$$

where the time constant $T_{t}$ and the damping coefficient $\xi_{t}$ are determined by the formulas

$$
T_{t}=\sqrt{M_{2} /\left(C_{e}+C_{33}^{E}\right)}, \xi_{t}=\alpha \delta^{2} C_{33}^{E} /\left(3 c^{E} \sqrt{M\left(C_{e}+C_{33}^{E}\right)}\right) .
$$

Let us consider the operation at low frequencies for the piezoactuator from piezoceramics PZT with one face rigidly fixed and elastic inertial load so that $M_{1} \rightarrow \infty$ and $m \ll M_{2}$ for $M_{2}=22.5 \mathrm{~kg}, C_{33}=2.3 \cdot 10^{6} \mathrm{~N} / \mathrm{m}$, $C_{e}=0.2 \cdot 10^{6} \mathrm{~N} / \mathrm{m}$ we obtain $T_{t}=3 \cdot 10^{-3} \mathrm{C}$. The experimental and calculated values for the piezoactuator are in agreement to an accuracy of $5 \%$.

\section{RESULTS AND Discussions}

From decision wave equation we obtain the generalized parametric structural schematic diagram of the piezoactuator of mechatronic systems for nanotechnology and adaptive optics on Figure 5, taking into account equation of piezoelectric effect. The results of constructing the generalized structural-parametric model and the generalized parametric structural schematic diagram of the piezoactuator for longitudinal, transverse and shift deformations are shown in Figure 5. Parametric structural schematic diagrams piezoactuator for longitudinal, transverse, shift piezoelectric effects Figure 2, Figure 3, Figure 4 converts to the generalized parametric structural schematic diagram of the piezoactuator Figure 5 with the replacement of the following parameters $\Psi_{m}=E_{3}, E_{3}, E_{1}, \quad v_{m i}=d_{33}, d_{31}, d_{15}, s_{i j}^{\Psi}=s_{33}^{E}, s_{11}^{E}, s_{55}^{E}, l=\delta, h, b$. 
Generalized structural-parametric model and generalized parametric structural schematic diagram of the piezoactuator after algebraic transformations provides the transfer functions of the piezoactuator for nanotechnology and adaptive optics. The piezoactuator with the transverse piezoelectric effect compared to the piezoelectric actuator for the longitudinal piezoelectric effect provides the greater range of static displacement and the less working force.

It is possible to construct the generalized structural-parametric model, generalized parametric structural schematic diagram and the transfer functions in matrix form of the piezoactuator using the solutions of the wave equation of the piezoactuator and taking into account the features of the deformations piezoactuator along the coordinate axes.

\section{ConcLusions}

Decision of wave equation, structural-parametric model, parametric structural schematic diagram, transfer functions of the piezoelectric actuator are obtained using the Laplace transform. The transfer functions and the parametric structural schematic diagram of the piezoactuator for the transverse, longitudinal, shift piezoelectric effects are obtained from the structural-parametric model of the piezoactuator. The transfer functions in matrix form are describe deformations of the piezoactuator during its operation as a part of the mechatronic systems for nanotechnology and adaptive optics.

The structural-parametric model of the piezoactuator describes its structure and conversion the energy electric field into mechanical energy and the corresponding displacements and forces at its the ends in contrast electrical equivalent circuits for calculation of piezoelectric transmitter and receiver. Piezoactuators are used for nanotechnology and adaptive optics, astronomy for large compound telescopes, antennas satellite telescopes for precise alignment, compensation of temperature and gravitational deformations and compensation of atmospheric turbulence.

Using the obtained decision of the wave equation and taking into account the features of the deformations along the coordinate axes are constructed the generalized structural-parametric model and the generalized parametric structural schematic diagram of the piezoactuator for nanotechnology and adaptive optics and are described its dynamic and static properties.

\section{REFERENCES}

1. Schultz J., Ueda J., Asada H. Cellular Actuators. Oxford: Butterworth-Heinemann Publisher, 382 p., 2017.

2. K. Uchino K. Piezoelectric actuator and ultrasonic motors. Boston, MA: Kluwer Academic Publisher, 347 p., 1997

3. Przybylski J. Static and dynamic analysis of a flextensional transducer with an axial piezoelectric actuation. Engineering structures. 2015; 84: 140-151, doi: 10.1016/j.engstruct.2014.11.025.

4. Ueda J., Secord T., Asada H. H. Large effective-strain piezoelectric actuators using nested cellular architecture with exponential strain amplification mechanisms. IEEE/ASME Transactions on Mechatronics. 2010; 15(5): 770-782, doi:10.1109/TMECH.2009.2034973.

5. Karpelson M., Wei G.-Y., R.J. Wood R.J. Driving high voltage piezoelectric actuators in microrobotic applications. Sensors and Actuators A: Physical. 2012: 176: 78-89, doi: 10.1016/j.sna.2011.11.035.

6. Gu G.-Y., Yang M.-J., L.-M. Zhu L.-M. Real-time inverse hysteresis compensation of piezoelectric actuators with a modified Prandtl-Ishlinskii model. Review of scientific instruments. 2012; 83(6): 065106, doi: $10.1063 / 1.4728575$. 
7. Afonin S.M. Solution of the wave equation for the control of an elecromagnetoelastic transduser. Doklady mathematics. 2006; 73(2): 307-313, doi:10.1134/S1064562406020402.

8. Afonin S.M. Structural parametric model of a piezoelectric nanodisplacement transduser. Doklady physics. 2008; 53(3): 137-143, doi:10.1134/S1028335808030063.

9. Afonin S.M. Stability of strain control systems of nano-and microdisplacement piezotransducers. Mechanics of solids. 2014; 49(2): 196-207, doi:10.3103/S0025654414020095.

10. Talakokula V., Bhalla S., Ball R.J., Bowen C.R., Pesce G.L., Kurchania R., Bhattacharjee B., Gupta A., Paine K. Diagnosis of carbonation induced corrosion initiation and progressionin reinforced concrete structures using piezo-impedance transducers. Sensors and Actuators A: Physical. 2016; 242: 79-91, doi: 10.1016/j. sna.2016.02.033.

11. Cady W.G. Piezoelectricity: An introduction to the theory and applications of electromechancial phenomena in crystals. New York, London: McGraw-Hill Book Company, 806 p., 1946.

12. Mason W., editor. Physical Acoustics: Principles and Methods. Vol.1. Part A. Methods and Devices. New York: Academic Press. 515 p., 1964.

13. Yang Y., Tang L. Equivalent circuit modeling of piezoelectric energy harvesters. Journal of intelligent material systems and structures. 2009; 20(18): 2223-2235.

14. Zwillinger D. Handbook of Differential Equations. Boston: Academic Press. 673 p., 1989.

15. Afonin S.M. Structural-parametric model and transfer functions of electroelastic actuator for nano- and microdisplacement. Chapter 9 in Piezoelectrics and Nanomaterials: Fundamentals, Developments and Applications. Parinov I.A., editor. New York: Nova Science. pp. 225-242, 2015.

16. Afonin S.M. Generalized parametric structural model of a compound elecromagnetoelastic transduser. Doklady physics. 2005; 50(2,): 77-82, doi:10.1134/1.1881716.

17. Afonin S.M. Parametric structural diagram of a piezoelectric converter. Mechanics of solids. 2002; 37(6): 85-91.

18. Afonin S.M. Deformation, fracture, and mechanical characteristics of a compound piezoelectric transducer. Mechanics of solids. 2003; 38(6): 78-82.

19. Afonin S.M. Parametric block diagram and transfer functions of a composite piezoelectric transducer. Mechanics of solids. 2004; 39(4): 119-127.

20. Afonin S.M. Elastic compliances and mechanical and adjusting characteristics of composite piezoelectric transducers. Mechanics of solids. 2007; 42(1): 43-49, doi:10.3103/S0025654407010062.

21. Afonin S.M. Static and dynamic characteristics of a multy-layer electroelastic solid. Mechanics of solids. 2009; 44(6): 935-950, doi:10.3103/S0025654409060119.

22. Afonin S.M. Design static and dynamic characteristics of a piezoelectric nanomicrotransducers. Mechanics of solids. 2010; 45(1): 123-132, doi:10.3103/S0025654410010152.

23. Afonin S.M. Structural-parametric model of nanometer-resolution piezomotor. Russian engineering research. 2001; 21(5): 42-50.

24. Afonin S.M. Parametric structure of composite nanometric piezomotor. Russian engineering research. 2002; 22(12): 9-24.

25. Afonin S.M. Electromechanical deformation and transformation of the energy of a nano-scale piezomotor. Russian engineering research. 2011; 31(7): 638-642, doi:10.3103/S1068798X11070033. 
Decision Wave Equation of Piezoactuator for Nanotechnology and Adaptive Optics

26. Afonin S.M. Electroelasticity problems for multilayer nano- and micromotors. Russian engineering research. 2011; 31(9): 842-847, doi:10.3103/S1068798X11090036.

27. Afonin S.M. Nano- and micro-scale piezomotors. Russian engineering research. 2012; 32(7-8): 519-522, doi:10.3103/S1068798X12060032.

28. Afonin S.M. Dynamic characteristics of multilayer piezoelectric nano- and micromotors. Russian engineering research. 2015; 35(2): 89-93, doi:10.3103/S1068798X15020045.

29. Afonin S.M. Optimal control of a multilayer submicromanipulator with a longitudinal piezo effect. Russian engineering research. 2015; 35(12): 907-910, doi:10.3103/S1068798X15120035.

30. Afonin S.M. Generalized structural parametric model of an elecromagnetoelastic transduser for control system of nano- and microdisplacement: I. Solution of the wave equation for control problem of an elecromagnetoelastic transduser. Journal of computer and systems sciences international. 2005; 44(3): 399-405.

31. Afonin S.M. Generalized structural parametric model of an elecromagnetoelastic transduser for control system of nano- and microdisplacements: II. On the generalized structural parametric model of a compound elecromagnetoelastic transduser. Journal of computer and systems sciences international. 2005; 44(4): 606-612.

32. Afonin S.M. Generalized structural-parametric model of an elecromagnetoelastic converter for nanoand micrometric movement control systems: III. Transformation parametric structural circuits of an elecromagnetoelastic converter for nano- and micromovement control systems. Journal of computer and systems sciences international. 2006; 45(2): 317-325, doi:10.1134/S106423070602016X.

33. Afonin S.M. Block diagrams of a multilayer piezoelectric motor for nano- and microdisplacements based on the transverse piezoeffect. Journal of computer and systems sciences international. 2015; 54(3): 424-439, doi:10.1134/S1064230715020021.

34. Afonin S.M. Absolute stability conditions for a system controlling the deformation of an elecromagnetoelastic transduser. Doklady mathematics. 2006; 74(3): 943-948, doi:10.1134/S1064562406060391.

35. Bhushan B., editor. Springer Handbook of Nanotechnology. New York: Springer, 1222 p., 2004.

36. Nalwa H. S., editor. Encyclopedia of Nanoscience and Nanotechnology. Calif.: American Scientific Publishers, 2004.

Citation: S.M. Afonin. "Decision Wave Equation of Piezoactuator for Nanotechnology and Adaptive Optics", American Research Journal of Physics, vol 3, no. 1, 2017, pp. 1-14.

Copyright (c) 2017 S.M. Afonin. This is an open access article distributed under the Creative Commons Attribution License, which permits unrestricted use, distribution, and reproduction in any medium, provided the original work is properly cited. 